\title{
In vivo repair of full-thickness cartilage defect with human iPSC-derived mesenchymal progenitor cells in a rabbit model
}

\author{
XINGQUAN XU ${ }^{1,2}$, DONGQUAN SHI ${ }^{1,2}$, YUBAO LIU ${ }^{1,2}$, YAO YAO $^{1}$, JIN DAI $^{1,2}$, \\ ZHIHONG XU ${ }^{1,2}$, DONGYANG CHEN ${ }^{1}$, HUAJIAN TENG ${ }^{2}$ and QING JIANG ${ }^{1,2}$ \\ ${ }^{1}$ Department of Sports Medicine and Adult Reconstructive Surgery, Drum Tower Hospital, School of Medicine, \\ Nanjing University, Nanjing, Jiangsu 210008; ${ }^{2}$ Joint Research Center for Bone and Joint Disease, \\ Model Animal Research Center (MARC), Nanjing University, Nanjing, Jiangsu 210093, P.R. China
}

Received July 26, 2015; Accepted January 20, 2017

DOI: $10.3892 / \mathrm{etm} .2017 .4474$

\begin{abstract}
Cell-based tissue engineering has the potential to restore cartilage defects. Induced pluripotent stem cells (iPSCs) are regarded as an alternative cell source in regenerative medicine. The purpose of the present study was to evaluate the use of mesenchymal stem cells (MSCs) derived from human iPSCs (hiPSCs) for the regeneration of cartilage defects in a rabbit model. Cartilage defects were made in the patellar grooves of New Zealand white rabbits. The rabbits were then divided into three groups according to implantation: Control group, scaffold implantation group and scaffold/hiPSCs-MSCs (experimental) group. MSCs were generated from hiPSCs via a step of embryoid body formation. Following flow cytological analysis, the hiPSCs-MSCs were plated onto poly(lactic-co-glycolide) and then transplanted into the cartilage defects in the experimental group. Six rabbits from each group were sacrificed at each time point. The outcome was assessed macroscopically and histologically at 3 and 6 weeks post-surgery. At 3 and 6 weeks, the experimental group showed more cartilage defect filling compared with the control and scaffold implantation groups. At 3 weeks, the experimental group showed much more repair tissue in the cartilage defect, although no cartilage-like tissue was observed. At 6 weeks, cartilage-like tissue was observed in the experimental group but not in the control or scaffold implantation groups. No teratoma formation was observed in any of the groups. The results indicate that iPSCs have the potential to repair cartilage defects in vivo. Therefore, iPSCs could be a new cell source for cartilage defect repair.
\end{abstract}

Correspondence to: Dr Qing Jiang or Dr Dongquan Shi, Department of Sports Medicine and Adult Reconstructive Surgery, Drum Tower Hospital, School of Medicine, Nanjing University, 321 Zhongshan Road, Nanjing, Jiangsu 210008, P.R. China

E-mail: qingj@nju.edu.cn

E-mail: shidongquan1215@163.com

Key words: tissue engineering, cartilage defect, regeneration, induced pluripotent stem cells, cell source

\section{Introduction}

Articular cartilage injury is increasing in incidence year by year, which is an important healthcare problem. Cell-based tissue engineering holds promise for restoring cartilage defects (1). To date, the most widely used cell sources in cartilage regeneration are mesenchymal stem cells (MSCs) and mature chondrocytes. Notably, MSCs have already been used to repair cartilage defects in clinical trials $(2,3)$. MSCs are easily obtained from various kinds of tissues, such as bone marrow, synovial tissue and muscle, and they would not be rejected by the immune system when used in vivo (4-6). However, the limited proliferation and differentiation potential has restrained the use of MSCs in regenerative medicine (7). In addition, the proliferative capability and differentiation potential of MSCs has been reported to decline with age (8).

Induced pluripotent stem cells (iPSCs) can be generated from well-differentiated somatic cells by introducing defined reprogramming transcription factors using retroviruses (9). iPSCs possess pluripotency, proliferation ability and multi-lineage differentiation potential similar to embryonic stem cells (ESCs) (9-11). In addition, a variety of new methods have been developed to generate iPSCs for the purpose of reducing the risk of tumor formation $(12,13)$. Therefore, iPSCs are regarded as alternative cell sources in regenerative medicine.

Undifferentiated iPSCs will form teratoma in vivo (14), which is the main obstacle to the use of iPSCs for tissue regeneration. The differentiation of iPSCs into MSCs has the promise to solve this problem. ESC markers (for example, Nanog and Sox2) were reported to no longer appear in iPSC-derived mesenchymal stem cells (iPSCs-MSCs), which may reduce the risk of tumorigenicity when used in vivo $(15,16)$. Studies have also showed that it is possible to induce the differentiation of iPSCs-MSCs into osteogenic, chondrogenic and vascular lineages in vitro (15-19). However, few studies have used iPSCs or iPSCs-MSCs to repair cartilage defects in vivo.

In the present study, mesenchymal progenitor cells were obtained from human iPSCs (hiPSCs) via embryoid body (EB) formation, a step that mimics embryonic development. The in vivo ability of hiPSCs-MSCs to repair cartilage defects was examined using a full-thickness cartilage defect rabbit model. 


\section{Materials and methods}

hiPSC culture. The hiPSC line (no. 0209-001; Sidan Sai Biotechnology Co., Ltd., Shanghai, China) was generated previously by introducing six reprogramming factors (Oct $3 / 4$, Sox2, Klf4, c-Myc, Nanog and Lin 28) into human newborn foreskin fibroblasts (20). The undifferentiated hiPSCs were maintained and expanded according to previous reported methods (20). In brief, chemically inactivated murine embryonic fibroblasts (MEFs) were used as feeder cells and were seeded on Matrigel-coated (Sigma-Aldrich; Merck Millipore, Darmstadt, Germany) dishes. hiPSCs were cultured on MEF feeder layers in ES medium (Sidan Sai Biotechnology Co., Ltd.) supplemented with $4 \mathrm{ng} / \mathrm{ml}$ basic fibroblast growth factor (bFGF) (Peprotech, Inc., Rocky Hill, NJ, USA). The medium was refreshed every day. Type IV collagenase (Sigma-Aldrich; Merck Millipore) was used to perform cell passage.

hiPSCs-MSCs preparation. Undifferentiated hiPSCs were detached from culture dishes using $1 \mathrm{mg} / \mathrm{ml}$ type IV collagenase and were then plated onto low-attachment culture dishes at a density of 1,000-1,200 cell clusters per $100 \mathrm{~mm}$ dish. The cells were allowed to aggregate and form spheres in a humidified atmosphere at $37^{\circ} \mathrm{C}$ and $5 \% \mathrm{CO}_{2}$ (Thermo Fisher Scientific, Inc., Waltham, MA, USA) in a maintenance medium containing Dulbecco's modified Eagle's medium (DMEM)/F12 and 10\% fetal bovine serum (FBS; Invitrogen; Thermo Fisher Scientific, Inc.). EBs formed after 7 days' suspension culture and were transferred to gelatin-coated (Sigma-Aldrich; Merck Millipore) dishes at $800-1,000 \mathrm{EBs} / 100 \mathrm{~mm}$ dish in expansion medium with DMEM/F12, 10\% FBS, $100 \mathrm{U} / \mathrm{ml}$ penicillin, and $100 \mathrm{mg} / \mathrm{m}^{2}$ streptomycin (all from Invitrogen; Thermo Fisher Scientific, Inc.). The cells sprouted from EBs were harvested as hiPSC-MSCs and expanded in expansion medium. The hiPSC-MSCs were purified by removing non-adherent cells.

Flow cytometry. The hiPSCs-MSCs at passage 3 were harvested. One million cells were suspended in $100 \mu \mathrm{l}$ buffer that consisted of $0.5 \%$ bovine serum albumin (BSA; Sigma-Aldrich; Merck Millipore) and 2 mM EDTA (Sunshine Biotechnology Co., Ltd., Nanjing, China). Subsequently, $10 \mu 1$ 1:10 diluted fluorescein isothiocyanate (FITC)-coupled antibodies recognizing CD11b (130-098-778), CD105 (130-098-778), CD90 (130-097-930), CD45 (130-098-043) and CD34 (130-098-142) (MACS; Miltenyi Biotec, Bergisch Gladbach, Germany) were added. In addition, 1:10 diluted mouse IgG1 (130-104-562) and mouse IgG2a (no. 130-098-877) antibodies (MACS; Miltenyi Biotec) were used as isotype controls. Incubation for $10 \mathrm{~min}$ incubation in the dark at $4^{\circ} \mathrm{C}$ was performed. The cells were then washed with buffer containing phosphate-buffered saline, $\mathrm{pH} 7.2$, $0.5 \%$ BSA, and 2 mM EDTA by diluting MACS BSA Stock Solution (130-091-376) 1:20 with autoMACS Rinsing Solution (130-091-222) (MACS; Miltenyi Biotec). Then, the cells were centrifuged at $300 \mathrm{x} \mathrm{g}$ for $10 \mathrm{~min}$ at $4^{\circ} \mathrm{C}$ and resuspended in $500 \mu \mathrm{l}$ of the aforementioned buffer for analysis by flow cytometry (BD FACSCalibur, BD Biosciences, Franklin Lakes, NJ, USA). The data was analyzed using Flowjo 7.6 sofrware (BD Biosciences).
Animal model and transplantation procedure. A total of 36 skeletally mature female New Zealand white rabbits (age, 12 weeks; weight, $2.0-2.5 \mathrm{~kg}$ ), purchased from the Jinling Farm, Nanjing, China were used in this study. Rabbits were fed a regular diet twice a day and allowed free access to water. They were housed under controlled conditions (temperature, $25 \pm 3^{\circ} \mathrm{C}$; humidity, $45 \pm 5 \%$; 12 -h light/dark cycle). All surgical procedures were approved by the Institutional Rabbit Care and Use Committee of Drum Tower Hospital, Medical School of Nanjing University (Nanjing, China). A full-thickness cartilage defect model was made in the trochlear grooves of the rabbits as previously reported (21). In brief, the rabbits were anesthetized with an intramuscular injection of $20 \mathrm{mg} / \mathrm{ml}$ xylazine hydrochloride (Huamu Animal Health Care Co., Ltd., Jilin, China) at a dose of $3 \mathrm{ml} / \mathrm{kg}$. The knee articular surface of the rabbits was exposed through a medial parapatellar approach. Whether the right or the left knee was used to perform the surgery was determined randomly. An osteochondral transplantation system ( $3.5 \mathrm{~mm}$ in diameter, $3.0 \mathrm{~mm}$ in depth) was used to create osteochondral defects. The rabbits were then divided into three groups according to implantation: Control group, scaffold implantation group and scaffold/hiPSCs-MSCs (experimental) group ( $\mathrm{n}=12$ per group).

The poly(lactic-co-glycolide) (PLGA) scaffold was purchased from Shandong Institute of Medical Instruments (Jinan, China). The average pore diameter of the PLGA scaffold was $\sim 200 \mu \mathrm{m}$. The PLGA scaffold was cut to $3.5 \times 3.5 \times 3 \mathrm{~mm}$ dimensions with a razor blade. The prepared PLGA scaffolds were immersed in Matrigel for $24 \mathrm{~h}$ to enhance cell attachment. Then, $5 \times 10^{6}$ hiPSCs-MSCs were seeded onto the prepared scaffold. After incubating in complete medium for $12 \mathrm{~h}$, the PLGA/hiPSCs-MSCs complex was transplanted into the cartilage defect in the experimental group. The scaffold implantation group received only PLGA scaffold, and the control group was untreated. Six rabbits from each group were sacrificed at 3 and 6 weeks after surgery. The repair quality was evaluated by gross and histological examination.

Histological analysis. The specimens were cut into 5- $\mu \mathrm{m}$ sections and stained with hematoxylin and eosin (H\&E) and toluidine blue as previous reported (21). In brief, the specimens were fixed, decalcified, dehydrated and embedded in paraffin. The specimens were then cut into $5-\mu \mathrm{m}$ sections and stained with H\&E (Beyotime Institute of Biotechnology, Shanghai, China) and toluidine blue (Toyond Biotechnology Co., Ltd., Shanghai, China) staining according to the manufacturers' instructions. The results were assessed independently by 3 different investigators.

\section{Results}

Generation of hiPSCs-MSCs. A multistep culture method consisting of spontaneous differentiation via a step of EB formation, cell outgrowth from EBs, and monolayer culture following cell dissociation was used in the present study to generate hiPSC-MSCs (Fig. 1). hiPSC-MSCs originated from the mesoderm and neural crest of the EBs and exhibited a spindle-like shape (Fig. 1D). Flow cytometric analysis was used to analyze the mesenchymal properties of the hiPSC-MSCs obtained in this study. The results showed that the majority of 

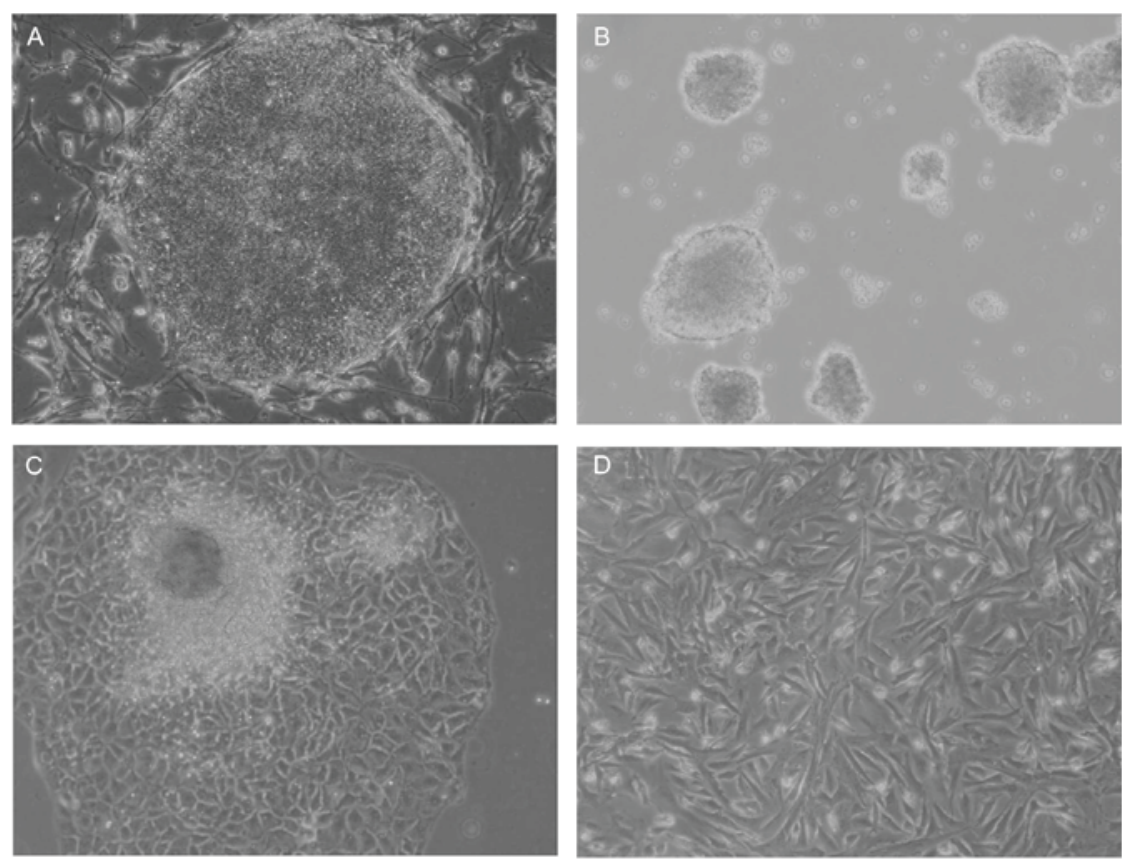

Figure 1. Generation of hiPSCs-MSCs. (A) Undifferentiated hiPSCs were cultured in human embryonic stem cell medium. (B) EB formation was observed after 7 days of suspension culture. (C) Cells sprouted out from EBs in culture medium containing Dulbecco's modified Eagle's medium/F12 and 10\% fetal bovine serum. (D) hiPSCs-MSCs exhibited spindle-like morphology. hiPSCs, human induced pluripotent stem cells; MSCs, mesenchymal stem cells; EB, embryoid body.
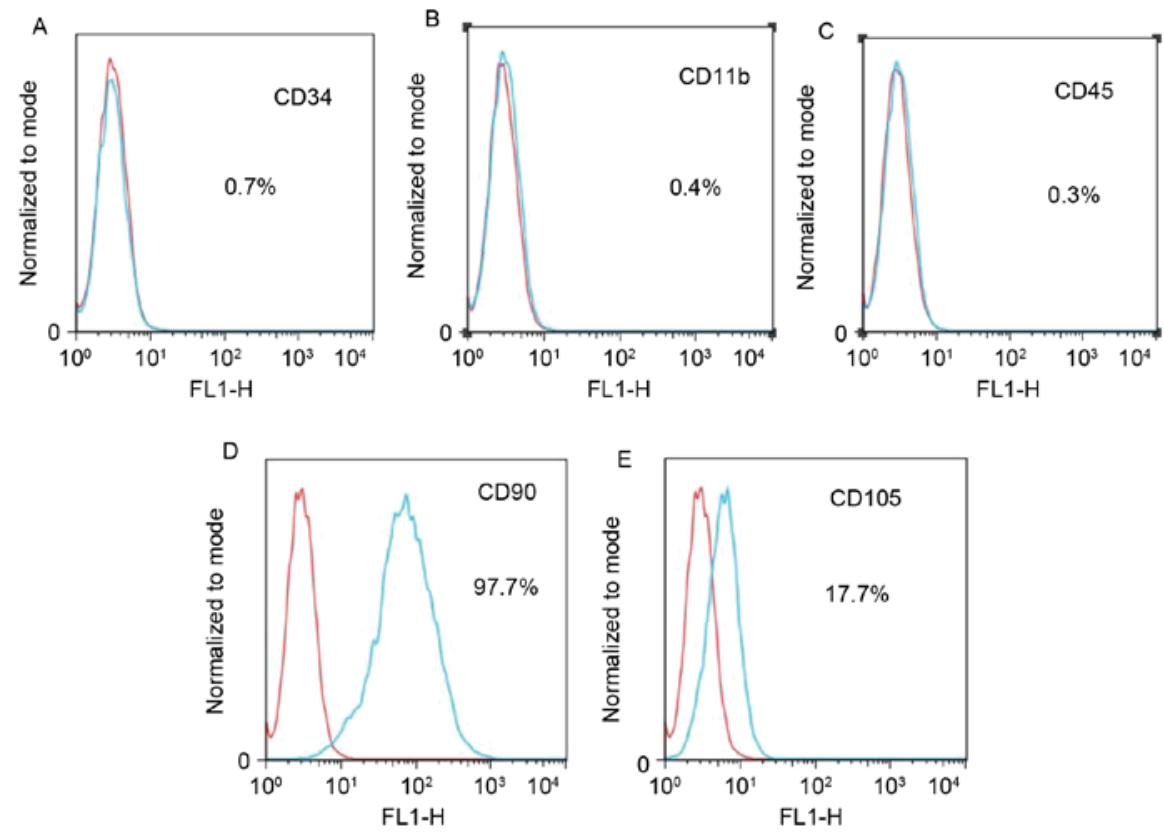

Figure 2. Flow cytometric analysis of human induced pluripotent stem cells-mesenchymal stem cells. (A) CD34, (B) CD11b, (C) CD45, (D) CD90 and (E) CD105 expression is shown as the green plots and isotype control expression as the red plots.

cells expressed CD90, and some expressed CD105, but most cells did not express CD34, CD11b or CD45 (Fig. 2).

Macroscopic evaluation of repair quality. Following transplantation (Fig. 3A), in the control and scaffold implantation groups, little repair tissue was observed in the cartilage defect 3 weeks after surgery (Fig. 3B and C). However, in the experimental group, repair tissue covering $>50 \%$ of the defects was observed (Fig. 3D). At 6 weeks, the cartilage defect was only partially covered by fibrous tissue in the control and scaffold implantation groups (Fig. 3E and F). At 6 weeks, repair tissue almost $100 \%$ filled the cartilage defect in the experimental group (Fig. 3G).

Histological evaluation of the repair quality. $\mathrm{H} \& \mathrm{E}$ staining showed better repair quality in the experimental group compared with that in the other two groups (Fig. 4). Only fibrous tissue was observed in defects of the control and 

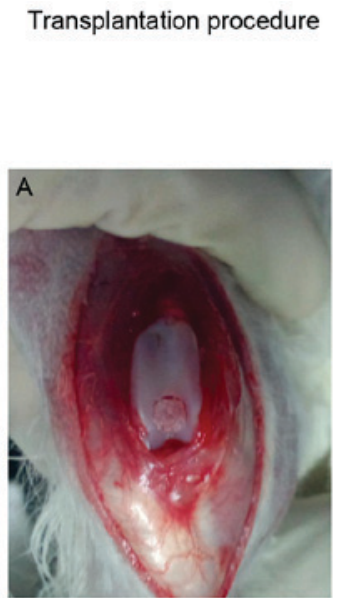

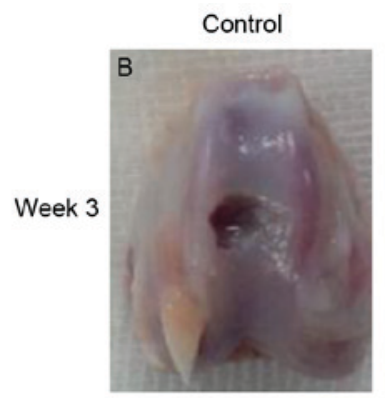

Week 6

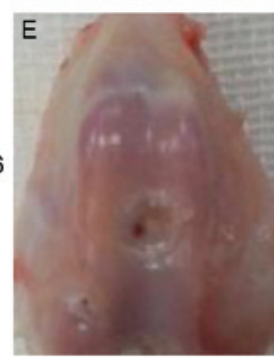

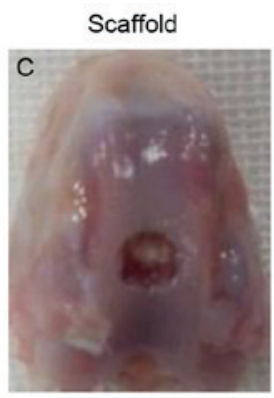

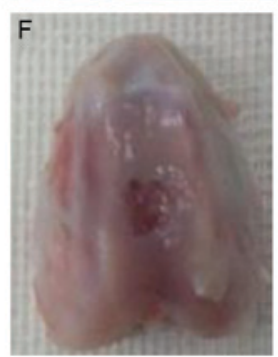

Experimental
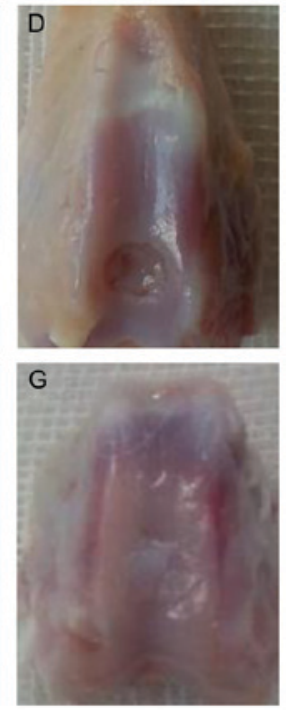

Figure 3. (A) Transplantation procedure for rabbit cartilage defects. The cartilage defect was left untreated or transplanted with scaffold or scaffold/hiPSCs-MSCs. Little repair tissue was observed in the cartilage defect in the (B) control and (C) scaffold only groups at 3 weeks. (D) However, in the scaffold/hiPSCs-MSCs group, repair tissue covered $>50 \%$ of the defect at 3 weeks. At 6 weeks, the cartilage defects were only partially covered by fibrous tissue in the (E) control and (F) scaffold only group. while (G) the cartilage defect was almost completely repaired in the scaffold/hiPSCs-MSCs group. Rabbits transplanted with scaffold/hiPSCs-MSCs formed the experimental group. hiPSCs, human induced pluripotent stem cells; MSCs, mesenchymal stem cells.
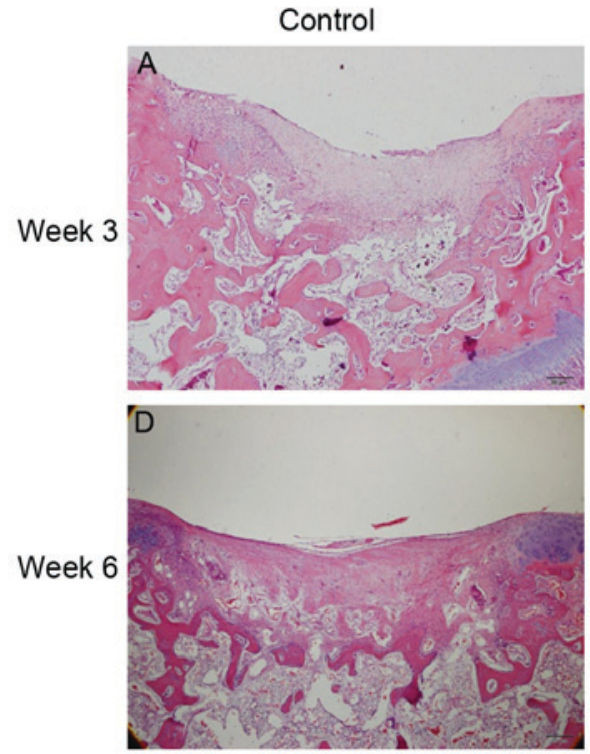
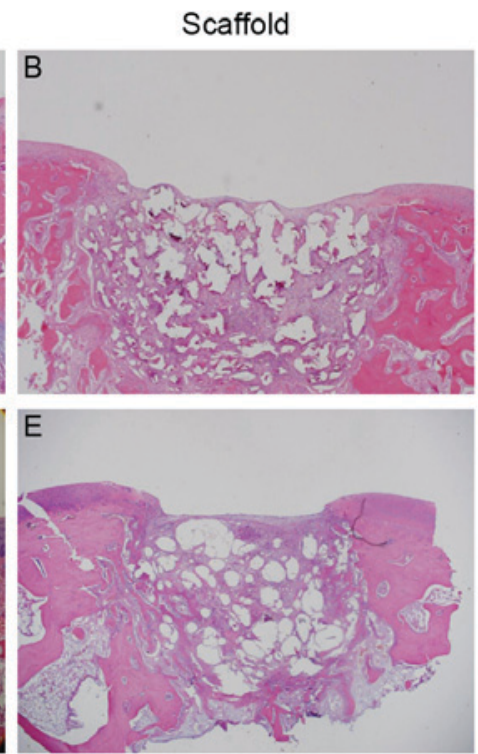
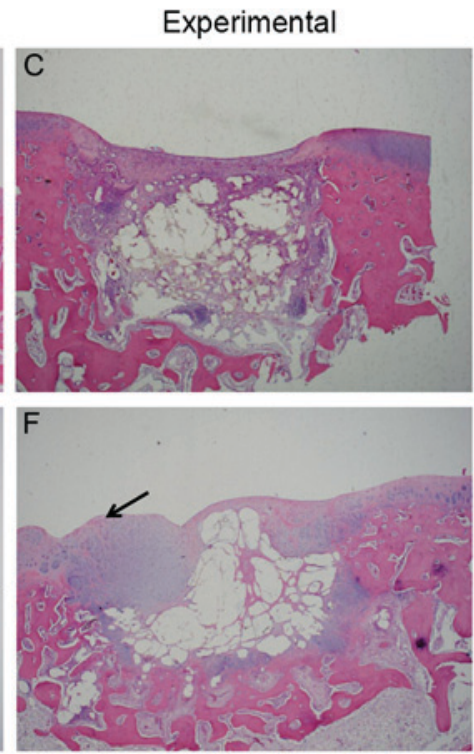

Figure 4. Representative hematoxylin and eosin staining of the sections at (A-C) 3 weeks and (D-F) 6 weeks after cartilage defect formation and treatment. Magnification, x20. At both 3 and 6 weeks, little fibrous tissue was observed in defects of the (A and D) control and (B and E) scaffold only groups. (C) At 3 weeks, a relative thicker repair tissue was observed in the top layer of cartilage defect in the scaffold/hiPSCs-MSCs group. (F) Cartilage-like tissue (arrow) was visible in the top layer of the defect at 6 weeks in the scaffold/hiPSCs-MSCs group. Subchondral bone formation was not observed in any of the groups. Rabbits transplanted with scaffold/hiPSCs-MSCs formed the experimental group. hiPSCs, human induced pluripotent stem cells; MSCs, mesenchymal stem cells.

scaffold implantation groups at 3 and 6 weeks. In the experimental group, H\&E staining showed cartilage-like tissue in the top layer of the defect at 6 weeks. However, subchondral bone formation was poor in all the groups. The newly formed tissue was stained slightly in the control and scaffold implantation groups at 6 weeks by toluidine blue staining (Fig. 5A-D). The matrix of regenerated tissue in the top layer of the defect in the experimental group was stained intensely. However, native cartilage degeneration was also observed (Fig. 5E and F).

\section{Discussion}

The results revealed cartilage-like tissue formation in the top layer of the cartilage defect when hiPSCs-MSCs were used. An apparently better quality of in vivo cartilage defect repair in the experimental group compared with the control and scaffold implantation groups was demonstrated by gross and histological appearance. Another important finding was that there was no evidence of teratoma formation in the 

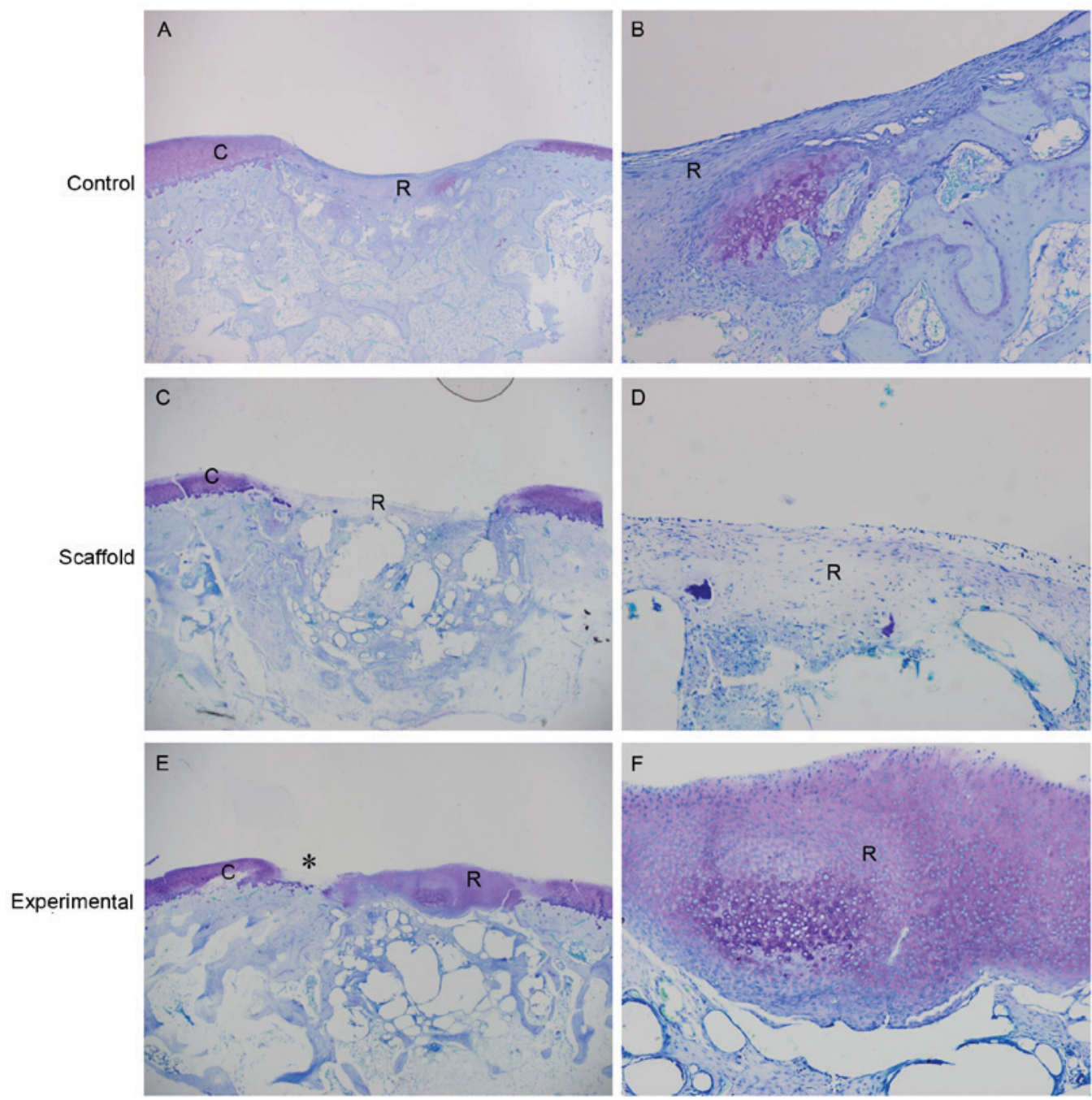

Figure 5. Representative toluidine blue staining of the sections at 6 weeks. The cartilage defect was poorly repaired in the control group at (A) low and (B) high magnification and scaffold only group at (C) low and (D) high magnification. At high magnification, in the control and scaffold only groups, the repair tissue was stained slightly and no cartilage-like tissue was observed. (E) In the experimental group, the repair tissue in the top layer of the cartilage defect was stained intensely, similar to native cartilage. Native cartilage degeneration was also observed (indicated by asterisk). At high magnification (F), cartilage-like tissue was observed in the top layer of the cartilage defect. (A, C and E) Magnification, x20. (B, D and F) Magnification, x100. Rabbits transplanted with scaffold/human induced pluripotent stem cells-mesenchymal stem cells formed the experimental group. R, repair tissue; C, cartilage.

experimental group. Although the restoration of full-thickness cartilage defect was not totally satisfactory, the results of the present study indicated that iPSCs may be a new cell source for cartilage defect repair in vivo.

iPSCs have been considered as the optimal cell source for regenerative medicine because of their self-renewal and pluripotency capability (22). Few studies have examined in vivo cartilage defect repair using iPSCs. Ko et al (19) reported successful induction of chondrogenesis and repair of cartilage defect in vivo with hiPSCs in immunosuppressed rats. Yamashita et al (23) reported hyaline chondrogenesis from hiPSCs and showed neo-cartilage survival in joint surface defects following newly generated cartilage particle transplantation in immunosuppressed rats and mini pigs. The results of the present study appear to be inferior to those of the aforementioned studies. This might be the result of using hiPSCs-MSCs transplantation, rather than newly generated cartilage transplantation in the present study, as local environmental inductive effects would be inferior to those of exogenous growth factors. Similarly, Marquass et al (24) also demonstrated that differentiated MSCs showed better histological outcomes compared with undifferentiated MSCs. However, the rabbit model used in the present study was more appropriate for the examination of cartilage defect repair than a rat model, as the cartilage thickness of rats is much thinner and the endogenous healing potential in rats is greater (25).

No teratoma formation was observed in the present study. This suggests that iPSCs-MSCs may be safer than iPSCs when used in vivo, although the mechanism is not clear. In previous studies, Ko et al (19) and Yamashita et al (23) did not report teratoma formation in vivo, consistent with observations in the present study. The method used to get hiPSCs-MSCs in the present study consisted of three steps: i) EB formation; ii) cell outgrowth from EBs; and iii) monolayer cell culture to select cells that can adapt to MSC growth conditions. Numerous alternative approaches for the preparation of MSCs from ESCs or iPSCs, such as using co-culture methods $(26,27)$, gene transfection (28) or conditioned medium (29) have been reported. However, the use of other cells or exogenous genetic material may introduce contamination with animal pathogens 
or the risk of tumorigenicity. Thus, the culture protocol used in the present study, which is simple and reproducible, appears to be suitable for the generation of MSCs from hiPSCs.

This study also had some limitations. Firstly, no examination was conducted to confirm whether the newly generated repair tissue was induced from transplanted hiPSCs-MSCs, or whether the implanted hiPSCs-MSCs remained in situ. Some unexpected factors may play a role during cartilage defect repair in vivo with hiPSCs-MSCs. It is possible that the paracrine effect of implanted hiPSCs-MSCs contributed to the attraction of host chondrocytes and MSCs to the cartilage defects. Second, the follow-up period may have limited the repair quality in this study. Results were only observed at 3 and 6 weeks, as we were keen to avoid any rejection reactions in the xenotransplantation model used in this study. There have been a few studies concerning xenotransplantation for cartilage defect repair. Pei et al (30) demonstrated failure of xenoimplantation using porcine MSCs for rabbit cartilage defects at a follow up of 6 months. However, Jang et al (31) reported a successful result in xenoimplantation of human MSCs into rabbit cartilage defects at 4 and 8 weeks. Thus, although there is no consensus for the appropriate follow-up period in xenoimplantation, the follow-up period in the present study may have been too short to induce rejection reactions. Thirdly, the hiPSCs-MSCs were not purified by cell sorting, which may also limit the cartilage defect repair.

Although this study had some limitations, it suggested that full-thickness cartilage defects can be repaired using hiPSCs-MSCs. Further understanding of the differentiation of iPSCs and a long-term investigation of full-thickness cartilage defect regeneration with iPSCs are necessary.

\section{Acknowledgements}

The present study was supported by the Projects of International Cooperation and Exchanges Natural Science Foundation of China (NSFC) (grant no. 81420108021), National Key Technology Support Program (grant no. 2015BAI08B02), Excellent Young Scholars NSFC (grant no. 81622033), NSFC (grant no. 81572129), Jiangsu Provincial Key Medical Center Foundation, Jiangsu Provincial Medical Talent Foundation and Jiangsu Provincial Medical Outstanding Talent Foundation, Social Development Project of Jiangsu Provincial Science and Technology Department (grant no. BE2016609).

\section{References}

1. Caplan AI: Review: Mesenchymal stem cells: Cell-based reconstructive therapy in orthopedics. Tissue Eng 11: 1198-1211, 2005

2. Wakitani S, Okabe T, Horibe S, Mitsuoka T, Saito M, Koyama T, Nawata M, Tensho K, Kato H, Uematsu K, et al: Safety of autologous bone marrow-derived mesenchymal stem cell transplantation for cartilage repair in 41 patients with 45 joints followed for up to 11 years and 5 months. J Tissue Eng Regen Med 5: 146-150, 2011

3. Wakitani S, Nawata M, Tensho K, Okabe T, Machida H and Ohgushi $\mathrm{H}$ : Repair of articular cartilage defects in the patello-femoral joint with autologous bone marrow mesenchymal cell transplantation: Three case reports involving nine defects in five knees. J Tissue Eng Regen Med 1: 74-79, 2007.

4. Segawa Y, Muneta T, Makino H, Nimura A, Mochizuki T, Ju YJ, Ezura Y, Umezawa A and Sekiya I: Mesenchymal stem cells derived from synovium, meniscus, anterior cruciate ligament and articular chondrocytes share similar gene expression profiles. J Orthop Res 27: 435-441, 2009.
5. Zuk PA, Zhu M, Ashjian P, De Ugarte DA, Huang JI, Mizuno H, Alfonso ZC, Fraser JK, Benhaim P and Hedrick MH: Human adipose tissue is a source of multipotent stem cells. Mol Biol Cell 13: 4279-4295, 2002.

6. Pittenger MF, Mackay AM, Beck SC, Jaiswal RK, Douglas R, Mosca JD, Moorman MA, Simonetti DW, Craig S and Marshak DR: Multilineage potential of adult human mesenchymal stem cells. Science 284: 143-147, 1999.

7. Im GI, Kim DY, Shin JH, Hyun CW and Cho WH: Repair of cartilage defect in the rabbit with cultured mesenchymal stem cells from bone marrow. J Bone Joint Surg Br 83: 289-294, 2001.

8. Payne KA, Didiano DM and Chu CR: Donor sex and age influence the chondrogenic potential of human femoral bone marrow stem cells. Osteoarthritis Cartilage 18: 705-713, 2010.

9. Takahashi K, Tanabe K, Ohnuki M, Narita M, Ichisaka T, Tomoda $\mathrm{K}$ and Yamanaka S: Induction of pluripotent stem cells from adult human fibroblasts by defined factors. Cell 131: 861-872, 2007.

10. Takahashi K and Yamanaka S: Induction of pluripotent stem cells from mouse embryonic and adult fibroblast cultures by defined factors. Cell 126: 663-676, 2006.

11. Wernig M, Meissner A, Foreman R, Brambrink T, Ku M, Hochedlinger $\mathrm{K}$, Bernstein $\mathrm{BE}$ and Jaenisch R: In vitro reprogramming of fibroblasts into a pluripotent ES-cell-like state. Nature 448: 318-324, 2007.

12. Kimura T, Kaga Y, Sekita Y, Fujikawa K, Nakatani T, Odamoto M, Funaki S, Ikawa M, Abe K and Nakano T: Pluripotent stem cells induced from mouse somatic cells by small-molecule compounds. Stem Cells 33: 45-55, 2015.

13. Lin T and Wu S: Reprogramming with small molecules instead of exogenous transcription factors. Stem Cells Int 2015: 794632, 2015.

14. Imaizumi M, Nomoto Y, Sato Y, Sugino T, Miyake M, Wada I, Nakamura T and Omori K: Evaluation of the use of induced pluripotent stem cells (iPSCs) for the regeneration of tracheal cartilage. Cell Transplant 22: 341-353, 2013.

15. Teramura T, Onodera Y, Mihara T, Hosoi Y, Hamanishi C and Fukuda K: Induction of mesenchymal progenitor cells with chondrogenic property from mouse-induced pluripotent stem cells. Cell Reprogram 12: 249-261, 2010.

16. Lian Q, Zhang Y, Zhang J, Zhang HK, Wu X, Zhang Y, Lam FF, Kang S, Xia JC, Lai WH, et al: Functional mesenchymal stem cells derived from human induced pluripotent stem cells attenuate limb ischemia in mice. Circulation 121: 1113-1123, 2010.

17. de Peppo GM, Marcos-Campos I, Kahlter DJ, Alsalman D, Shang L, Vunjak-Novakovic G and Marolt D: Engineering bone tissue substitutes from human induced pluripotent stem cells. Proc Natl Acad Sci USA 110: 8680-8685, 2013.

18. Diekman BO, Christoforou N, Willard VP, Sun H, Sanchez-Adams J, Leong KW and Guilak F: Cartilage tissue engineering using differentiated and purified induced pluripotent stem cells. Proc Natl Acad Sci USA 109: 19172-19177, 2012.

19. Ko JY, Kim KI, Park S and Im GI: In vitro chondrogenesis and in vivo repair of osteochondral defect with human induced pluripotent stem cells. Biomaterials 35: 3571-3581, 2014.

20. Liao J, Wu Z, Wang Y, Cheng L, Cui C, Gao Y, Chen T, Rao L, Chen S, Jia N, et al: Enhanced efficiency of generating induced pluripotent stem (iPS) cells from human somatic cells by a combination of six transcription factors. Cell Res 18: 600-603, 2008.

21. Xu X, Shi D, Shen Y, Xu Z, Dai J, Chen D, Teng H and Jiang Q: Full-thickness cartilage defects are repaired via a microfracture technique and intraarticular injection of the small-molecule compound kartogenin. Arthritis Res Ther 17: 20, 2015.

22. Ng KK, Thatte HS and Spector M: Chondrogenic differentiation of adult mesenchymal stem cells and embryonic cells in collagen scaffolds. J Biomed Mater Res A 99: 275-282, 2011.

23. Yamashita A, Morioka M, Yahara Y, Okada M, Kobayashi T, Kuriyama S, Matsuda S and Tsumaki N: Generation of scaffoldless hyaline cartilaginous tissue from human iPSCs. Stem Cell Reports 4: 404-418, 2015.

24. Marquass B, Schulz R, Hepp P, Zscharnack M, Aigner T, Schmidt S, Stein F, Richter R, Osterhoff G, Aust G, et al: Matrix-associated implantation of predifferentiated mesenchymal stem cells versus articular chondrocytes: In vivo results of cartilage repair after 1 year. Am J Sports Med 39: 1401-1412, 2011.

25. Chu CR, Szczodry M and Bruno S: Animal models for cartilage regeneration and repair. Tissue Eng Part B Rev 16: 105-115, 2010.

26. Barberi T, Willis LM, Socci ND and Studer L: Derivation of multipotent mesenchymal precursors from human embryonic stem cells. PLoS Med 2: e161, 2005. 
27. Bigdeli N, Karlsson C, Strehl R, Concaro S, Hyllner J and Lindahl A: Coculture of human embryonic stem cells and human articular chondrocytes results in significantly altered phenotype and improved chondrogenic differentiation. Stem Cells 27: $1812-1821,2009$

28. Xu C, Jiang J, Sottile V, McWhir J, Lebkowski J and Carpenter MK: Immortalized fibroblast-like cells derived from human embryonic stem cells support undifferentiated cell growth. Stem Cells 22: 972-980, 2004.

29. Hwang YS, Polak JM and Mantalaris A: In vitro direct chondrogenesis of murine embryonic stem cells by bypassing embryoid body formation. Stem Cells Dev 17: 971-978, 2008.
30. Pei M, Yan Z, Shoukry M and Boyce BM: Failure of xenoimplantation using porcine synovium-derived stem cell-based cartilage tissue constructs for the repair of rabbit osteochondral defects. J Orthop Res 28: 1064-1070, 2010.

31. Jang KM, Lee JH, Park CM, Song HR and Wang JH: Xenotransplantation of human mesenchymal stem cells for repair of osteochondral defects in rabbits using osteochondral biphasic composite constructs. Knee Surg Sports Traumatol Arthrosc 22: 1434-1444, 2014. 